\title{
The Effect of a Subnormal Vitamin B-6 Status on Homocysteine Metabolism
}

\author{
Johan B. Ubbink, ${ }^{\star}$ Annatjie van der Merwe, ${ }^{*}$ Rhena Delport, ${ }^{\star}$ Robert H. Allen, ${ }^{\ddagger}$ Sally P. Stabler, ${ }^{\ddagger}$ Reiner Riezler, $\$$ \\ and \\ *Department of Chemical Pathology, University of Pretoria, Pretoria 0001, South Africa; ${ }^{\ddagger}$ Division of Hematology, University of \\ Colorado Health Center, Denver, Colorado 80262; and ${ }^{\S}$ Severimed, 48163 Münster, Germany
}

\begin{abstract}
Homocysteine, an atherogenic amino acid, is either remethylated to methionine or metabolized to cysteine by the transsulfuration pathway. The biochemical conversion of homocysteine to cysteine is dependent upon two consecutive, vitamin B-6-dependent reactions.

To study the effect of a selective vitamin B-6 deficiency on transsulfuration, we performed oral methionine load tests on 22 vitamin B-6-deficient asthma patients treated with theophylline (a vitamin B-6 antagonist) and 24 age- and sex-matched controls with a normal vitamin B-6 status. Both groups had normal circulating vitamin B-12 and folate concentrations. Methionine loading resulted in significantly higher increases in circulating total homocyst(e)ine $(P<0.01)$ and cystathionine $(P<0.05)$ concentrations in vitamin B-6-deficient patients compared with controls. $6 \mathrm{wk}$ of vitamin B-6 supplementation $(20 \mathrm{mg} / \mathrm{d})$ significantly $(P<0.05)$ reduced post-methionine load increases in circulating total homocyst(e)ine concentrations in deficient subjects, but had no significant effect on the increase in total homocyst(e)ine concentrations in controls. The increases in post-methionine load circulating cystathionine concentrations were significantly $(P<0.01)$ reduced in both groups after vitamin supplementation.
\end{abstract}

It is concluded that a vitamin B-6 deficiency may contribute to impaired transsulfuration and an abnormal methionine load test, which is associated with premature vascular disease. (J. Clin. Invest. 1996. 98:177-184.) Key words: folate - methionine - coronary heart disease $\cdot$ vitamin B-12 • cystathionine

\section{Introduction}

Aberrant homocysteine metabolism has been associated with divergent clinical conditions such as premature vascular diseases $(1-6)$ and neural tube defects $(7,8)$. Several factors may impede effective homocysteine metabolism. Genetic factors, such as homozygosity for cystathionine- $\beta$-synthase $\left(\mathrm{CBS}^{1}\right.$; EC

Address correspondence to Johan B. Ubbink, Department of Chemical Pathology, University of Pretoria, P.O. Box 2034, Pretoria 0001, South Africa. Phone: 27-12-3192550; FAX: 27-12-3283600; E-mail: jubbink@medic.up.ac.za

Received for publication 12 December 1995 and accepted in revised form 28 March 1996.

1. Abbreviations used in this paper: CBS, cystathionine- $\beta$-synthase; MTHFR, 5,10-methylenetetrahydrofolate reductase; PLP, pyridoxal 5 '-phosphate; tHcy, total homocyst(e)ine.

J. Clin. Invest.

(C) The American Society for Clinical Investigation, Inc.

0021-9738/96/07/177/08 \$2.00

Volume 98, Number 1, July 1996, 177-184
4.2.1.22) deficiency or 5,10-methylenetetrahydrofolate reductase (MTHFR; EC 1.7.99.5) deficiency, may result in severe hyperhomocyst(e)inemia ${ }^{2}$ with serious clinical sequelae, e.g., advanced atherosclerosis of major arteries and/or vascular thrombosis at an early age (9-11). Milder impairment of enzymes involved in homocysteine metabolism is associated with an increased risk for neural tube defect pregnancies (12) and premature vascular diseases (13-15). Nutritional factors are also important determinants of homocysteine metabolism. The metabolism of homocysteine to cysteine is catalyzed by two vitamin B-6-dependent enzymes, i.e., CBS, which catalyzes the condensation of homocysteine with serine to form cystathionine, and cystathionase (EC 4.4.1.1), which catalyzes the hydrolysis of cystathionine to cysteine and $\alpha$-ketobutyrate (Fig. 1). Remethylation of homocysteine requires vitamin B-12 and methyltetrahydrofolate as coenzyme and cosubstrate, respectively (16). Therefore, a suboptimal vitamin status is associated with elevated fasting plasma total homocyst(e)ine (tHcy) concentrations (17-20) and with an increased risk for vascular stenosis and coronary heart disease $(5,19)$.

Mild impairment of homocysteine remethylation is characterized by elevated, fasting plasma tHcy concentrations, irrespective of whether the impairment is nutritionally (folate or vitamin B-12 deficiency [21-23]) or genetically (heat labile MTHFR [24, 25]) induced. However, partially impaired transsulfuration in heterozygotes for CBS deficiency is often not associated with elevated fasting plasma tHcy concentrations (2628). When the transsulfuration pathway is stressed by an oral L-methionine load test, it is sometimes possible to differentiate between heterozygotes and controls by the higher postmethionine load plasma tHcy concentrations in the former group $(27,28)$. In addition, changes in vitamin B-6 status do not affect fasting plasma tHcy concentrations $(29,30)$, while high dose pyridoxine supplementation has been shown to attenuate the post-methionine load plasma tHcy concentrations $(31,32)$.

Little is known about whether a vitamin B-6 deficiency may contribute to an abnormal methionine load test. Given that $S$-adenosylmethionine is an MTHFR inhibitor and a CBS activator, Selhub and Miller postulated that a vitamin B-6 deficiency will result in post-methionine load hyperhomocyst(e)inemia (33). According to Selhub and Miller's hypothesis, the high post-methionine load intracellular $S$-adenosylmethionine concentrations will inhibit homocysteine remethylation, while activation of transsulfuration cannot proceed because of the vitamin B-6 deficiency (33). Although earlier studies indicated increased urinary homocyst(e)ine excretion in vitamin B-6-deficient subjects after methionine loading $(34,35)$, a later study did not find a significant relation between plasma pyridoxal $5^{\prime}$ phosphate (PLP) and the post-methionine load tHcy concen-

2. Total homocyst(e)ine refers to the sum of the concentrations of free homocysteine, protein-bound homocysteine, the disulfide homocystine, and the mixed disulfide homocysteine-cysteine. 


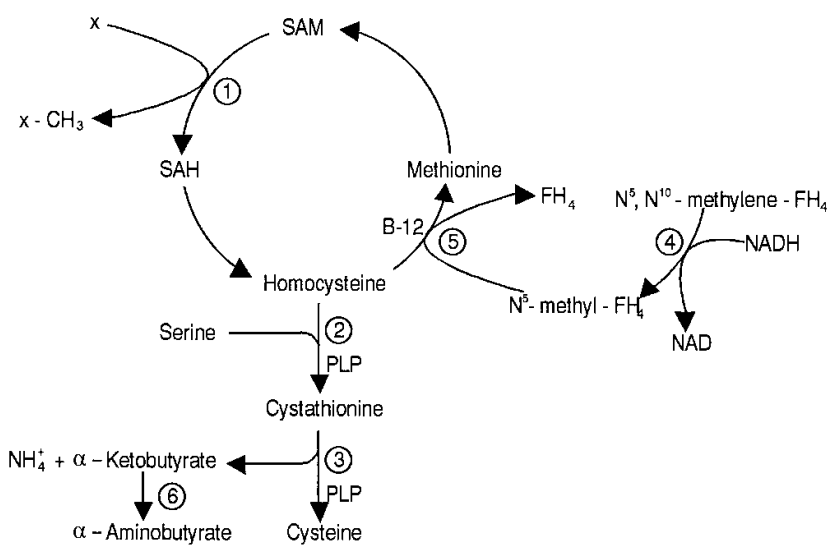

Figure 1. Methionine and homocysteine metabolism. Homocysteine is either remethylated to methionine or metabolized by the transsulfuration pathway. Enzymes: 1, various methyltransferases; 2, CBS; 3, cystathionase; 4, MTHFR; 5, 5-methyltetrahydrofolate homocysteine methyltransferase; 6 , transaminase(s). X, general methyl group acceptor; $\mathrm{FH}_{4}$, methyltetrahydrofolate.

trations in 72 patients with vascular disease (31). Recent studies on vitamin B-6-deficient rats found significantly elevated post-methionine load plasma tHcy concentrations in deficient animals compared with controls $(36,37)$. It is not known whether a suboptimal vitamin B-6 status in free-living human subjects may contribute to aberrant transsulfuration and increased vascular disease risk.

Due to the inhibition $(38,39)$ of the enzyme pyridoxal kinase (EC 2.7.1.35), chronic treatment with theophylline results in a vitamin B-6 deficiency as characterized by low circulating PLP concentrations $(38,40)$, severely reduced erythrocyte transaminase activities (40) and abnormal tryptophan metabolism (38), while the folic acid and vitamin B-12 status remain unaffected. Since theophylline-treated asthmatics are a good example of free-living individuals with a selective vitamin B-6 deficiency, we used this group to investigate the effect of $(a)$ a vitamin B-6 deficiency, and $(b)$ pyridoxine supplementation on the transsulfuration pathway when stressed by the oral methionine load test.

\section{Methods}

Subject selection. Asthma patients and controls were invited to participate in the trial through the local press. Volunteers who phoned the trial coordinator were questioned to determine whether they met the preliminary inclusion criteria. Prospective participants had to be $>21 \mathrm{yr}$ of age without any other life-threatening conditions such as diabetes, severe liver disorders, coagulation disorders, renal insufficiency, or alcohol/drug abuse. They were excluded if they reported treatment with vitamin $\mathrm{K}$ antagonists, intravenous heparin, or vitamin B-containing supplements 3 mo before the study entry. Asthma patients had to be on theophylline therapy.

Volunteers who fulfilled the criteria mentioned above were invited to the laboratory (visit 0 ) where a $10-\mathrm{ml}$ clotted blood sample was obtained for a clinical chemistry profile, while blood samples with EDTA as anticoagulant were obtained for a full blood count as well as measurement of plasma vitamin and theophylline concentrations. All blood samples were obtained after an overnight fast, and, in the case of asthma patients, before the morning dose of theophylline was taken. The following inclusion criteria had to be fulfilled to enter a patient with asthma into the trial: $(a)$ plasma PLP $<32.0 \mathrm{nmol} / \mathrm{liter}$, (b) plasma trough theophylline concentration $>5.0 \mathrm{mg} / \mathrm{dl}$, (c) serum alanine aminotransferase $<35 \mathrm{IU} /$ liter, $(d)$ serum aspartate aminotransferase $<35 \mathrm{IU} /$ liter, $(e)$ serum gamma-glutamyl transferase $<46 \mathrm{IU} / \mathrm{liter},(f)$ serum creatinine $<140 \mu \mathrm{mol} / \mathrm{liter}$, and $(g)$ blood platelet count $>150,00010^{9} /$ liter.

Controls were apparently healthy, age- (within $5 \mathrm{yr}$ ) and sexmatched volunteers who complied with the same exclusion/inclusion criteria as described above, with two exceptions, i.e., plasma PLP concentration $>35 \mathrm{nmol} /$ liter and the plasma theophylline concentration had to be below the detection limit of the applied analytical method.

Volunteers who fulfilled the criteria as outlined above gave informed consent before they were allowed to enter the study. 27 asthmatics (10 men and 17 women) and 26 controls ( 8 men and 18 women) were enrolled into the trial. The study was approved by the University Human Ethics Committee and was performed according to the guidelines of the Declaration of Helsinki.

Treatment of participants. Enrolled participants visited the laboratory consulting rooms three times, each time after an overnight fast. During visit 1 , a complete physical examination was performed by a qualified, registered clinician. The following parameters were recorded: body weight, height, radial pulse, and systolic and diastolic blood pressures. Medical histories and use of medication were also recorded. Following blood sampling ( $10 \mathrm{ml}$ clotted blood sample, 10 $\mathrm{ml}$ blood with EDTA as anticoagulant), L-methionine $(100 \mathrm{mg} / \mathrm{kg}$ body weight) dissolved in $250 \mathrm{ml}$ of orange juice was administered. Blood samples were again obtained 4,6 , and $8 \mathrm{~h}$ after methionine administration. $2 \mathrm{~h}$ after methionine administration, a light, methionine-poor meal was served to the participants. After completion of the methionine load test, the participants received pyridoxine tablets (sugar-coated lactose-based tablets, containing $10.0 \mathrm{mg}$ of pyridoxine, prepared by Vesta Medicines, Johannesburg, South Africa) and were instructed to take two tablets daily after dinner. An appointment was made for visit 2 , scheduled to take place $3 \mathrm{wk}$ after visit 1.

At visit 2, clotted blood as well as EDTA blood samples were obtained to monitor vitamin status and the participant received a further 3-wk supply of pyridoxine tablets. Visit 3 took place 3 wk after visit 2; during this visit the participant again reported for a methionine load test after an overnight fast. The methionine load test was performed as described above, followed by a complete medical examination.

At each visit, participants returned remaining pyridoxine tablets, which were counted to determine participant compliance. Concomitant asthma medication continued during the trial.

Laboratory analyses. EDTA plasma was used for vitamin and tHcy analysis. Blood samples with EDTA as anticoagulant were centrifuged within $1 \mathrm{~h}$ of collection to prevent artificial increases in plasma tHcy concentrations. Plasma samples were stored at $-70^{\circ} \mathrm{C}$ for vitamin and tHcy analyses after completion of the trial. This allowed batching of samples in order to minimize day-to-day analytical variation. Plasma vitamin and tHcy analyses were performed in duplicate; duplicates were always performed in different batches and on different days.

Plasma PLP was determined by HPLC as previously described (41). The day-to-day coefficient of variation for the two in-house controls was 11.5 and $8.4 \%$ for the low and normal controls, respectively. Plasma concentrations of vitamin B-12 and folate were determined by a commercially available radioassay kit (Becton Dickinson and Co., Orangeburg, NY).

Plasma homocyst(e)ine was derivatized with ammonium 7-fluorobenzo-2 oxa 1, 3 diazole-4-sulfonate (Wako, Neuss, Germany) according to the method of Araki and Sako (42). The method entails complete reduction of homocystine, the mixed disulphide (cysteinehomocysteine), and release of protein-bound homocysteine. Therefore, this method measures total (free plus protein-bound) plasma homocyst(e)ine concentrations. The SBD-derivative of homocysteine was determined by HPLC (43). The day-to-day variation of two inhouse control plasma samples was 8.4 and $8.2 \%$ for the normal and high controls, respectively. 
Table I. Vitamin Status of Study Participants

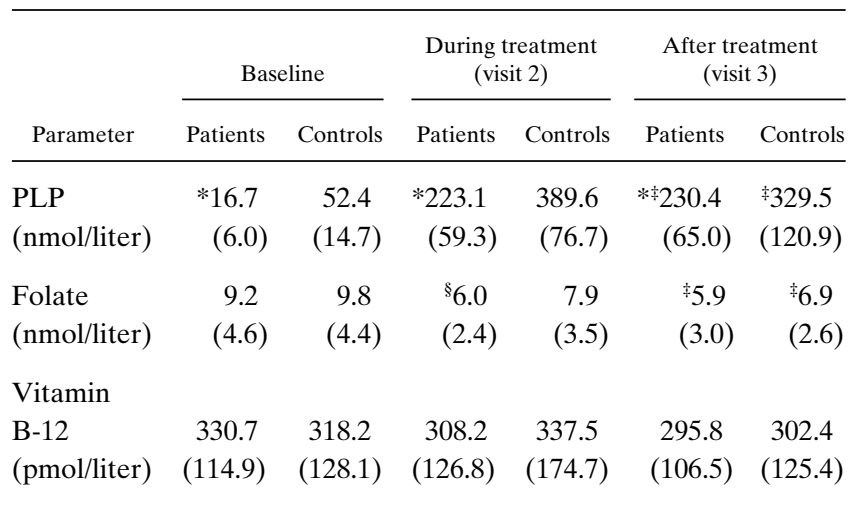

Results are expressed as mean (SD). ${ }^{*}$ Indicates significant betweengroup differences between patients and controls. ${ }^{*} P<0.001,{ }^{\S} P<0.05$. ${ }^{\ddagger}$ Indicates significant within-group differences when postpyridoxine supplementation concentrations (visit 3 ) were compared with baseline concentrations, $P<0.001$.

Plasma theophylline concentrations were measured by HPLC according to the method of Blanchard (44).

Serum samples were analyzed for metabolites involved in the transsulfuration pathway (45). The profile of metabolites included methionine, tHcy, cystathionine, serine, $\alpha$-aminobutyric acid, and cysteine. Methylmalonate and 2-methyl citrate were also measured as functional indices of vitamin B-12 status.

Statistical analyses. Generally, the data sets showed normal distributions and the two groups of participants who completed the study were therefore compared with Student's $t$ test, taking unequal variances into account (Levine's test) where necessary. Given the small groups, the results were also confirmed with the Mann-Whitney U test. Within-group comparisons were done with Student's paired $t$ test and confirmed by Wilcoxon's matched-paired signed ranks test (46).

\section{Results}

22 patients ( 8 men, 14 women) and 24 controls ( 8 men, 16 women) successfully completed the trial. The mean (SD) age of the patients was $51.2(12.5) \mathrm{yr}$, which was not significantly different from the 51.9 (12.7) yr mean age of the controls. Asthma patients and controls did not differ with respect to body mass [76.3 (16.2) vs. $74.7(13.6) \mathrm{kg}$, respectively]. The asthma patients had been treated with theophylline for a mean (SD) period of $17.6(11.3) \mathrm{yr}$.

Compliance, based on counts of returned tablets, was $96.8 \%$ for patients and $97.7 \%$ for controls. No clinically significant changes were observed in the clinical chemistry and hematology profiles during the study. Plasma trough theophylline concentrations did not change significantly during the study period (results not shown).

The averages of each of the fasting circulating vitamin and metabolite concentrations of visits 0 and 1 were considered as baseline concentrations for each participant. As expected, baseline PLP concentrations of asthma patients were significantly lower compared with controls (Table I). After pyridoxine supplementation, plasma PLP concentrations increased significantly in both groups, but PLP remained significantly lower in patients compared with controls. Plasma vitamin B-12 concentrations in the two groups were not significantly different during the study period (Table I), but the mean (SD) baseline serum methylmalonate concentration was significantly $(P<$ $0.05)$ higher in patients [173.3 (54.1) nmol/liter] compared with controls [136.3 (35.8) nmol/liter]. This difference remained during the study period and at the third visit patients had a mean (SD) serum methylmalonate concentration of 171.9 (65.4) compared with 126.5 (32.6) nmol/liter in controls $(P<$ $0.05)$. The mean (SD) serum 2-methylcitrate concentrations in patients and controls were, respectively, $136.6(40.5)$ and 135.3 (34.0) nmol/liter at the start of the study, while the respective concentrations were 153.4 (65.4) and 133.7 (37.2) nmol/liter at the end of the study. Neither of these between-group differences was statistically significant.

Plasma folate concentrations in the two groups were not significantly different at screening or at visit 1 , but declined significantly during pyridoxine supplementation in both groups (Table I). 10 asthma patients had suboptimal plasma folate concentrations $(<5.0 \mathrm{nmol} / \mathrm{liter})$ at the end of the pyridoxine

Table II. Fasting Concentrations of Metabolites Implicated in the Transsulfuration Pathway

\begin{tabular}{|c|c|c|c|c|c|c|}
\hline \multirow[b]{2}{*}{ Parameter } & \multicolumn{2}{|c|}{ Baseline } & \multicolumn{2}{|c|}{ During treatment (visit 2) } & \multicolumn{2}{|c|}{ After treatment (visit 3) } \\
\hline & Patients & Controls & Patients & Controls & Patients & Controls \\
\hline Methionine & 22.4 & 24.7 & 25.7 & 27.8 & 21.9 & 23.7 \\
\hline$(\mu \mathrm{mol} /$ liter $)$ & (5.3) & $(5.2)$ & (7.1) & $(7.0)$ & (3.4) & (3.8) \\
\hline Homocysteine & $* 9.7$ & 8.0 & $* 9.3$ & 8.2 & $* 9.9$ & 8.4 \\
\hline$(\mu \mathrm{mol} /$ liter $)$ & (3.8) & (1.9) & $(2.5)$ & (1.9) & (3.4) & $(2.0)$ \\
\hline Cystathionine & 0.288 & 0.201 & $* 0.243$ & 0.195 & $\S_{0.168}$ & $\S_{0.156}$ \\
\hline$(\mu \mathrm{mol} /$ liter $)$ & $(0.179)$ & $(0.048)$ & $(0.116)$ & $(0.059)$ & $(0.075)$ & $(0.042)$ \\
\hline Cysteine & 298.0 & 275.4 & 290.1 & 276.1 & $\|_{298.4}$ & 282.8 \\
\hline ( $\mu \mathrm{mol} /$ liter $)$ & $(42.8)$ & $(21.4)$ & $(41.2)$ & $(20.2)$ & $(42.5)$ & $(16.1)$ \\
\hline$\alpha$-Aminobutyrate & $\| 9.3$ & 15.1 & $\|_{11.6}$ & 19.4 & $\| 9.8$ & 16.9 \\
\hline ( $\mu \mathrm{mol} /$ liter $)$ & (3.7) & (4.4) & (4.7) & $(12.6)$ & $(5.0)$ & (6.1) \\
\hline Serine & 139.7 & 141.7 & 144.0 & 152.4 & 135.1 & 141.8 \\
\hline$(\mu \mathrm{mol} /$ liter $)$ & $(15.9)$ & (19.8) & $(24.5)$ & $(21.6)$ & $(13.8)$ & $(17.4)$ \\
\hline
\end{tabular}

Results are expressed as mean (SD). ${ }^{*}$ Between-group differences were marginally not significant $0.05>P<0.1$. ${ }^{*}$ Indicates significant betweengroup differences between patients and controls, ${ }^{\|} P<0.01$ and ${ }^{\ddagger} P<0.05$. ${ }^{\S}$ Indicates significant within-group differences when postpyridoxine supplementation concentrations (visit 3) were compared with baseline concentrations, $P<0.01$. 
Table III. The Effect of the Methionine Load Test on Metabolites Implicated in Transsulfuration

\begin{tabular}{|c|c|c|c|c|}
\hline \multirow[b]{2}{*}{ Parameter } & \multicolumn{2}{|c|}{$\begin{array}{c}\text { Before pyridoxine } \\
\text { supplementation (visit 1) }\end{array}$} & \multicolumn{2}{|c|}{$\begin{array}{c}\text { After pyridoxine } \\
\text { supplementation (visit 3) }\end{array}$} \\
\hline & Patients & Controls & Patients & Controls \\
\hline Methionine & $* 632.5$ & 556.2 & 612.2 & 583.0 \\
\hline$(\mu \mathrm{mol} /$ liter $)$ & (133.5) & (108.7) & $(127.5)$ & $(137.5)$ \\
\hline Homocysteine & $\$ 23.9$ & 16.2 & $* \$ 20.3$ & 15.7 \\
\hline ( $\mu \mathrm{mol} /$ liter $)$ & (11.3) & (3.8) & (9.3) & (4.9) \\
\hline Cystathionine & $* 2.213$ & 1.374 & $\$ 1.439$ & $\S_{0.984}$ \\
\hline ( $\mu \mathrm{mol} /$ liter $)$ & (1.402) & $(0.893)$ & $(0.631)$ & (0.399) \\
\hline Cysteine & -30.1 & -28.0 & -34.1 & -33.8 \\
\hline ( $\mu \mathrm{mol} /$ liter $)$ & $(24.8)$ & $(17.9)$ & $(21.4)$ & $(12.2)$ \\
\hline Serine & -24.4 & -23.7 & -16.3 & -16.3 \\
\hline ( $\mu \mathrm{mol} /$ /iter) & (20.4) & (30.3) & (9.9) & (9.3) \\
\hline
\end{tabular}

Results are expressed as mean (SD). The postmethionine load metabolite concentrations were corrected for basal levels and the results represent the mean maximum concentration change for each metabolite. ${ }^{* *}$ Indicates significant between-group differences between patients and controls, ${ }^{\ddagger} P<0.01,{ }^{*} P \leq 0.05$. ${ }^{\S}$ Indicates significant within-group differences when postpyridoxine supplementation concentrations (visit 3) were compared with prepyridoxine (visit) supplementation concentration, $P<0.05$.

supplementation period in comparison with only 4 patients before pyridoxine supplementation was started.

Circulating tHcy concentrations were first determined by the HPLC method of Ubbink et al. (43), and later repeated (as part of a comprehensive metabolite assay) by GC-MS according to the method of Allen et al. (45). The methods correlated well $(r=0.95)$, therefore, only the tHcy concentrations as measured by HPLC are reported.

The fasting concentrations of metabolites implicated in the transsulfuration pathway are summarized in Table II. Although patients had higher fasting plasma tHcy concentrations than controls, the differences were not statistically significant. Before pyridoxine supplementation, serum cystathionine concentrations were significantly higher in patients compared with controls $(P<0.05)$, but this difference disappeared upon vitamin B-6 supplementation. The significantly lower $\alpha$-aminobutyric acid concentrations in the patient group persisted throughout the study. Patients and controls did not differ with respect to serum concentrations of methionine, serine, and cysteine.

Within-group comparisons did not show any statistically significant changes in fasting metabolite concentrations, except for serum cystathionine concentrations, which declined significantly in both patients and controls during pyridoxine supplementation.

In the subgroup of 10 patients who sustained suboptimal plasma folate concentrations during the course of the study, the mean (SD) plasma tHcy concentration after pyridoxine supplementation was 10.3 (3.8) $\mu \mathrm{mol} / \mathrm{liter}$, which did not differ significantly from the mean (SD) baseline tHcy concentration of $9.9(5.0) \mu \mathrm{mol} / \mathrm{liter}$.

The effect of the methionine load test on metabolites involved in the transsulfuration pathway was evaluated before and after pyridoxine supplementation as follows. Post-methionine load metabolite concentrations were corrected for basal levels, i.e., the change in metabolite concentrations was com- puted for every post-methionine load measurement. We followed this procedure because we did not want to confound the effect of methionine loading by basal concentrations which differed significantly in the two groups. Subsequently, the mean maximum change (increase or decrease) for each metabolite was calculated (Table III) and patients and controls were compared with respect to maximum changes in circulating metabolite concentrations.

Comparing patients with controls, methionine loading resulted in significantly higher maximum increases in serum methionine $(P<0.05)$, plasma tHcy $(P<0.01)$, and serum cystathionine $(P<0.05)$ concentrations in patients. Compared with presupplementation values, repetition of the methionine load test after 6 wk of pyridoxine supplementation resulted in significantly lower maximum increases in plasma tHcy $(P<$ $0.05)$ and serum cystathionine $(P<0.01)$ concentrations in patients. In controls, the mean maximum increase in plasma tHcy concentrations was not affected by pyridoxine supplementation, but the mean maximum increase in cystathionine levels declined significantly $(P<0.05)$. Despite the effect of pyridoxine supplementation on above-mentioned metabolite concentrations, the significant differences between patients and controls with respect to maximum concentration changes persisted. The only exception was methionine, where post-load concentrations did not differ any more after pyridoxine supplementation.

By selecting the maximum change in each patient a bias could be introduced, therefore we also expressed the effect of methionine loading as a function of time $(\mu \mathrm{mol} / \mathrm{liter} \cdot \mathrm{h})$, i.e., the area under the concentration versus time graph after methionine loading. These areas were computed by using the trapezium rule for those metabolites which showed significant between-group differences with respect to maximum changes in post-methionine load metabolite concentrations.

When the increases in circulating methionine concentrations after methionine loading were expressed as $\mu \mathrm{mol} / \mathrm{liter} \cdot \mathrm{h}$ over the 8-h follow-up period, it was found that patients [mean $(\mathrm{SD})=3343.4(787.0) \mu \mathrm{mol} / \mathrm{liter} \cdot \mathrm{h}]$ and controls [2934.5 (674.0) $\mu \mathrm{mol} /$ liter $\cdot h]$ did not differ significantly. Mean (SD) post-pyridoxine supplementation increases in methionine levels after methionine loading were $3248.2(751.6) \mu \mathrm{mol} / \mathrm{liter} \cdot \mathrm{h}$ for patients and $3076.6(747.4) \mu \mathrm{mol} / \mathrm{liter} \cdot \mathrm{h}$ for controls; this difference was not statistically significant (Fig. 2). For the increase in plasma tHcy concentration, the mean (SD) response to methionine loading in patients over an 8-h period was 124.2 (61.6) $\mu \mathrm{mol} / \mathrm{liter} \cdot \mathrm{h}$, which was significantly higher than the 89.6 (22.8) $\mu \mathrm{mol} / \mathrm{liter} \cdot \mathrm{h}$ measured in controls $(P<0.05)$. Pyridoxine supplementation over 6 wk resulted in a significant decline to $107.1(44.9) \mu \mathrm{mol} / \mathrm{liter} \cdot \mathrm{h}(P<0.05)$ in patients, but the decline to $84.8(27.7) \mu \mathrm{mol} / \mathrm{liter} \cdot \mathrm{h}$ in controls was not statistically significant. The difference between plasma tHcy responses in controls and patients after methionine loading and pyridoxine supplementation was on the border of statistical significance $(P=0.05$; Fig. 3$)$. For the increase in serum cystathionine concentrations, the mean (SD) response to methionine loading in patients over an 8-h period was 11.5 (7.6) $\mu \mathrm{mol} / \mathrm{liter} \cdot \mathrm{h}$, which was significantly higher than the $7.7(5.1) \mu \mathrm{mol} / \mathrm{liter} \cdot \mathrm{h}$ measured in controls $(P<0.05)$. Pyridoxine supplementation over $6 \mathrm{wk}$ resulted in a significant decline to $7.7(3.5) \mu \mathrm{mol} / \mathrm{liter} \cdot \mathrm{h}$ $(P<0.01)$ in patients and to $5.4(2.4) \mu \mathrm{mol} / \mathrm{liter} \cdot \mathrm{h}$ in controls $(P<0.05)$. The difference between cystathionine responses in controls and patients after pyridoxine supplementation and 


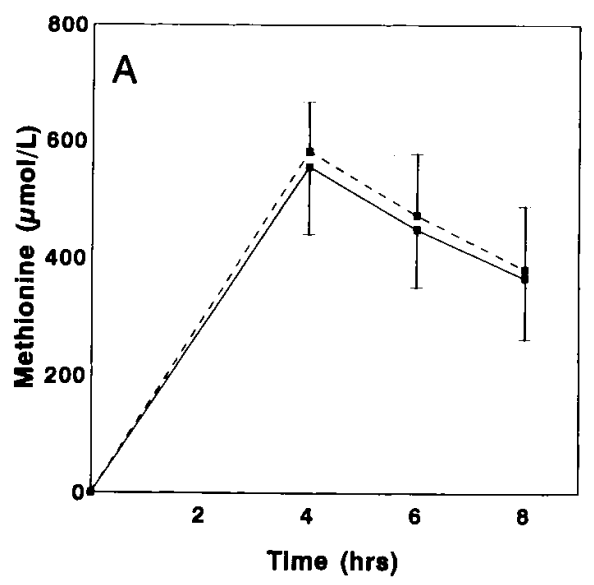

methionine loading remained statistically significant $(P<0.05$; Fig. 4).

\section{Discussion}

In view of the consistent association between elevated plasma tHcy concentrations and vascular disease (1-6), it has become imperative to define the optimum therapy to reduce circulating tHcy concentrations. Both folic acid and vitamin B-12 supplementation reduce fasting plasma tHcy concentrations (26, $30,47)$, but vitamin B-6 supplementation has no effect on basal tHcy levels $(30,31)$. Based on these results it may be argued that vitamin B-6 is superfluous in the treatment of moderate hyperhomocyst(e)inemia. However, a normal fasting tHcy concentration is not necessarily synonymous with normal homocysteine metabolism. Studies where both fasting and postmethionine load tHcy concentrations were measured indicate the existence of a subgroup of patients with normal fasting tHcy concentrations but an abnormal methionine load test (7, 48). In fact, the prevalence of post-methionine load hyperhomocyst(e)inemia in NHLBI Family Heart Study participants with normal fasting plasma tHcy concentrations was reported
Figure 2. The effect of the oral methionine load test on the increase in serum methionine concentrations before and after pyridoxine supplementation. The increase in serum methionine concentrations before (straight lines) and after (dashed lines) pyridoxine supplementation was plotted against time, and the areas under the curves (AUC) were computed by using the trapezium rule. Controls and patients did not differ with respect to the AUC. Pyridoxine supplementation had no effect on the mean AUC for both patients and controls. $A$, controls; $B$, patients. to be as high as $8.2 \%$ (49). Given that elevated post-methionine load tHcy concentrations are associated with both premature vascular disease $(1,2)$ and neural tube defects $(7)$, it seems prudent that vitamin therapy aimed at normalizing homocysteine metabolism should not only lower fasting tHcy concentrations, but also normalize the tHcy concentrations after oral methionine loading. The aim of this study is to investigate whether a vitamin B-6 deficiency in free-living humans may contribute to impaired transsulfuration as indicated by tHcy accumulation in the circulation after methionine loading. By using asthma patients treated with theophylline (a pyridoxal kinase antagonist), we were able to study the effect of a methionine load test in patients with vitamin B-6 deficiency in the presence of a normal folate and vitamin B-12 status (Table I).

A profile of fasting concentrations of metabolites from the transsulfuration pathway already indicates that transsulfuration is impeded during a theophylline-induced vitamin B-6 deficiency. Although the differences in fasting tHcy concentrations between patients and controls were not statistically significant, patients had significantly higher serum cystathionine concentrations (Table II). Cystathionine is a substrate for the PLPdependent enzyme cystathionase (EC 4.4.1.1), which metabo-
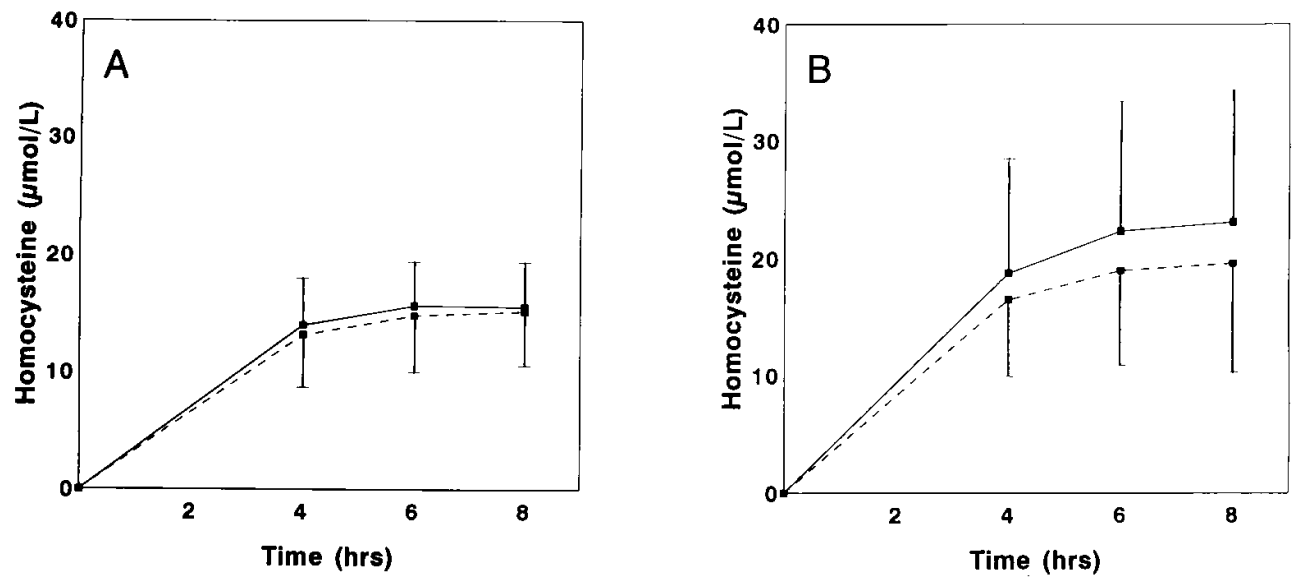

Figure 3. The effect of the oral methionine load test on the increase in plasma homocysteine concentrations before and after pyridoxine supplementation. The increase in plasma homocysteine concentrations before (straight lines) and after (dashed lines) pyridoxine supplementation was plotted against time, and the areas under the curves (AUC) were computed by using the trapezium rule. Before pyridoxine, the mean AUC was significantly higher in patients compared with controls $(P<0.05)$; pyridoxine supplementation resulted in a significant reduction in the mean AUC for patients $(P<0.05)$, but not for controls. $A$, controls; $B$, patients. 


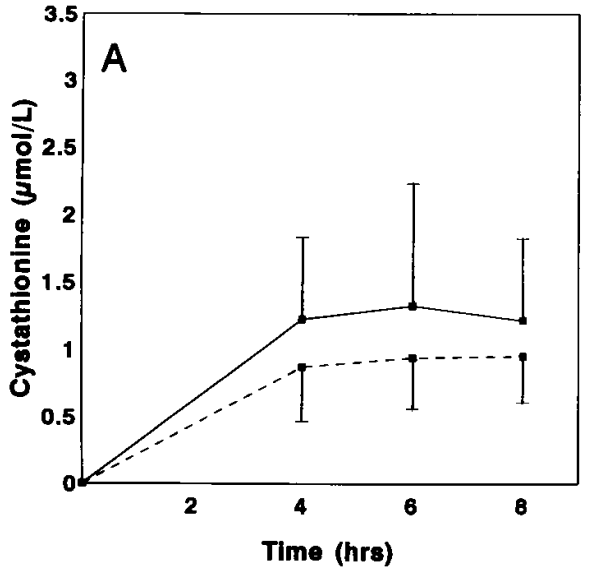

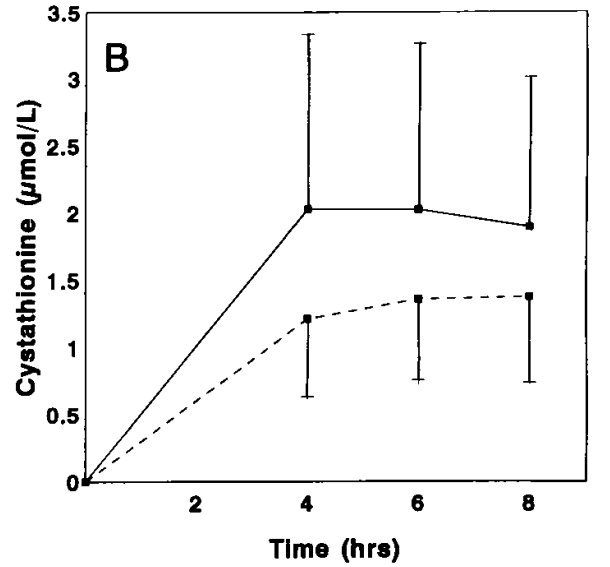

Figure 4. The effect of the methionine load test on the increase in serum cystathionine concentrations before and after pyridoxine supplementation. The increase in plasma cystathionine concentrations before (straight lines) and after (dashed lines) pyridoxine supplementation was plotted against time, and the areas under the curves (AUC) were computed by using the trapezium rule. Before pyridoxine, the mean AUC was significantly higher in patients compared with controls $(P<0.05)$; pyridoxine supplementation resulted in a significant reduction in the mean AUC for both patients $(P<$ $0.01)$ and controls $(P<0.05)$. $A$, controls; $B$, patients. lizes cystathionine to $\alpha$-ketobutyrate and cysteine in the second step of the transsulfuration pathway. This enzyme is known to be sensitive to vitamin B-6 depletion $(34,50)$ and the higher patient cystathionine concentrations before pyridoxine supplementation indicate reduced enzyme activity. However, even during a theophylline-induced vitamin B-6 deficiency, the PLP-dependent CBS activity is still sufficient to prevent accumulation of circulating tHcy under fasting conditions. Pyridoxine supplementation had no effect on fasting tHcy concentrations, but reduced serum cystathionine concentrations significantly in both patients and controls, thus indicating enhanced cystathionase activity in both groups. We have no explanation for the significant differences between patient and control $\alpha$-aminobutyrate (the transamination product of $\alpha$-ketobutyrate; Fig. 1) concentrations which persisted throughout the study (Table II).

After methionine loading, the increase in serum methionine concentrations before vitamin B-6 supplementation peaked at significantly higher levels in patients than in controls (Table III), but this difference disappeared after $6 \mathrm{wk}$ of vitamin B-6 supplementation. One possible interpretation would be that impaired transsulfuration resulted in methionine accumulation in the circulation, but a comparison of peak concentrations may introduce a positive bias into the data. The latter possibility is supported by the observation that the increase in serum methionine concentrations expressed as a function of time $(\mu \mathrm{mol} / \mathrm{liter} \cdot \mathrm{h})$ did not differ significantly between the two groups. There was no discrepancy between peak metabolite levels and metabolite levels expressed as a function of time for any of the other metabolites.

After methionine loading, the increases in circulating tHcy and cystathionine concentrations were significantly higher in the vitamin B-6-depleted patients than in controls, indicating impaired transsulfuration during a vitamin B-6 deficiency. The results imply that both the PLP-dependent condensation of serine with homocysteine to form cystathionine and the PLPdependent $\beta, \gamma$ elimination by cystathionase to yield cysteine and $\alpha$-ketobutyrate were partially inhibited in the patients. Vitamin B-6 supplementation resulted in significantly smaller increases in circulating tHcy and cystathionine concentrations in patients after methionine loading, indicating an enhanced capacity of the transsulfuration pathway. In controls, the increase in plasma tHcy concentrations did not change significantly when methionine loading followed the 6-wk pyridoxine supplementation period, indicating that pyridoxine supplementation did not enhance cystathionine $\beta$-synthase activity in individuals with a normal vitamin B-6 status. However, the increase in cystathionine concentrations after methionine loading was significantly attenuated by vitamin B-6 supplementation.

Our results indicate that although pyridoxine supplementation attenuates the post-methionine load increase in plasma tHcy concentrations in individuals with a vitamin B-6 deficiency, it has little effect in controls with a normal vitamin B-6 status. This contrasts with a previous study where vitamin (1 $\mathrm{mg}$ of folate, $0.4 \mathrm{mg}$ of vitamin B-12, $10 \mathrm{mg}$ of pyridoxine) supplementation resulted in a significant $38.3 \%$ decline in the mean post-methionine load increase in plasma tHcy concentration in young Caucasian men with plasma folate, vitamin B-12, and PLP concentrations within the normal reference range (51). Taken together, the results suggest that folate and/ or vitamin B-12 supplementation may lower the post-methionine load increase in plasma tHcy concentrations even in presence of a normal vitamin status, while pyridoxine supplementation will only affect the methionine load test in vitamin B-6-deficient individuals. The inference that folate and/or vitamin B-12 supplementation will reduce the increase in circulating post-methionine load tHcy concentrations is unexpected in view of MTHFR inhibition by $S$-adenosyl methionine (33). Further studies are required to define the effect of individual and combined vitamin supplements on post-methionine load plasma tHcy concentrations.

Although pyridoxine supplementation significantly lowered the patients' response to methionine loading with respect to the accumulation of homocysteine and cystathionine, these metabolite levels were nevertheless still higher than those observed in controls. This may be explained by the following. First, the period of pyridoxine supplementation was too short to correct the depressed vitamin B-6 status induced by a mean period of $17.6 \mathrm{yr}$ of theophylline therapy. Second, the daily pyridoxine supplement $(20 \mathrm{mg})$ might have been too small to 
reverse the metabolic effects of theophylline completely. It should be recognized that theophylline could have other effects on sulfur amino acid metabolism, e.g., interaction with PLP binding sites on CBS and cystathionase. Possible competition by theophylline for the above-mentioned PLP binding sites will be overcome by a higher, daily vitamin B-6 supplement. Third, it is also possible that vitamin B-6 supplementation may have compromised folate status (Table I). However, fasting plasma tHcy concentrations (a sensitive indicator of folate deficiency) did not increase significantly in the patient subgroup who sustained suboptimal plasma folate concentrations during pyridoxine supplementation. This indicates that the induced lower plasma folate concentrations may have little or no metabolic consequences. Finally, the possibility that subtle differences with respect to vitamin B-12 status may explain why pyridoxine supplementation failed to fully normalize transsulfuration in patients cannot be excluded. The mean serum methylmalonate concentration, a sensitive metabolic indicator of a depressed vitamin B-12 status (23), was significantly higher in patients compared with controls, however, the patient methylmalonate levels were still well within the normal range (73-271 $\mu \mathrm{mol} /$ liter; reference 23 ), indicating that the vitamin B-12 status was only marginally (if at all) affected. It is also possible that the higher serum methylmalonate concentrations in asthmatics are drug induced and not necessarily the result of a compromised vitamin B-12 status. This possibility is supported by the observation that serum 2-methylcitrate concentrations, which increase during a vitamin B-12 deficiency (52), did not differ significantly between controls and patients.

In conclusion, our results indicate that a subnormal vitamin B-6 status may result in methionine intolerance and impaired transsulfuration as characterized by accumulation of tHcy and cystathionine in the circulation after methionine loading. It is concluded that a vitamin B-6 deficiency may contribute to an abnormal methionine load test, which is an independent risk factor for premature vascular disease.

\section{Acknowledgments}

L. Goddard and A. Schnell provided excellent technical assistance.

The study has been supported by a grant from MEDICE, Iserlohn, Germany.

\section{References}

1. Clarke, R., L. Daly, K. Robinson, E. Naughten, S. Cahalane, B. Fowler, and I. Graham. 1991. Hyperhomocysteinemia: an independent risk factor for vascular disease. N. Engl. J. Med. 324:1149-1155.

2. Murphy-Chutorian, D.R., M.P. Wexman, A.J. Grieco, J.A. Heininger, E. Glassman, G. Gaull, F. Feit, K. Wexman, and A.C. Fox. 1985. Methionine intolerance: a possible risk factor for coronary artery disease. J. Am. Coll. Cardiol. 6: $725-730$

3. Malinow, M.R., J. Nieto, M. Szklo, L.E. Chambless, and G. Bond. 1993. Carotid artery intimal-medial wall thickening and plasma homocysteine in asymptomatic adults. Circulation. 87:1107-1113.

4. Wu, L.L., J. Wu, S.C. Hunt, B.C. James, G.M. Vincent, R.R. Williams, and P.N. Hopkins. 1994. Plasma homocyst(e)ine as a risk factor for early familial coronary artery disease. Clin. Chem. 40:552-561.

5. Selhub, J., P.F. Jacques, A.G. Bostom, R.B. D'Agostino, P.W.F. Wilson, A.J. Belanger, D.H. O'Leary, P.A. Wolf, E.J. Schaefer, and I.H. Rosenberg. 1995. Association between plasma homocysteine concentrations and extracranial carotid-artery stenosis. N. Engl. J. Med. 332:286-291.

6. Glueck, C.J., P. Show, J.E. Lang, T. Tracey, L. Sieve-Smith, and Y. Wang. 1995. Evidence that homocysteine is an independent risk factor for atherosclerosis in hyperlipidemic patients. Am. J. Cardiol. 75:132-136.

7. Steegers-Theunissen, R.P.M., G.H.J. Boers, F.J.M. Trijbels, J.D. Finkel- stein, H.J. Blom, C.M.G. Thomas, G.F. Borm, M.G.A.J. Wouters, and T.K.A.B. Eskes. 1994. Maternal hyperhomocysteinemia: a risk factor for neural-tube defects? Metab. Clin. Exp. 43:1475-1480.

8. Mills, J.L., J.M. McPartlin, P.N. Kirke, Y.J. Lee, M.R. Conley, D.G. Weir, and J.M. Scott. 1995. Homocysteine metabolism in pregnancies complicated by neural-tube defects. Lancet. 345:149-151.

9. Gibson, J.B., N.A.J. Carson, and D.W. Neill. 1964. Pathological findings in homocystinuria. J. Clin. Pathol. 17:427-437.

10. McCully, K.S. 1969. Vascular pathology of homocysteinemia: implications for the pathogenesis of arteriosclerosis. Am. J. Pathol. 56:111-128.

11. Kanwar, Y.S., J.R. Manaligod, and P.W.K. Wong. 1976. Morphologic studies in a patient with homocystinuria due to 5,10-methylenetetrahydrofolate reductase deficiency. Pediatr. Res. 10:598-609.

12. Van der Put, N.M.J., R.P.M. Steegers-Theunissen, P. Frosst, F.J.M. Trijbels, T.K.A.B. Eskes, L.P. van den Heuvel, E.C.M. Mariman, M. Den Heyer, R. Rozen, and H.J. Blom. 1995. Mutated methylenetetrahydrofolate reductase as a risk factor for spina bifida. Lancet. 346:1070-1071.

13. Boers, G.H.J., A.G.H. Smals, F.J.M. Trijbels, B. Fowler, J.A.J.M. Bakkeren, H.C. Schoonderwaldt, W.J. Kleijer, and P.W.C. Kloppenborg. 1985. Heterozygosity for homocystinuria in premature peripheral and cerebral occlusive arterial disease. N. Engl. J. Med. 313:709-715.

14. Kang, S.S., P.W.K. Wong, A. Susmano, J. Sora, M. Norusis, and N. Ruggie. 1991. Thermolabile methylenetetrahydrofolate reductase: an inherited risk factor for coronary artery disease. Am. J. Hum. Genet. 48:536-545.

15. Kang, S.S., E.L. Passen, N. Ruggie, P.W.K. Wong, and H. Sora. 1993. Thermolabile defect of methylenetetrahydrofolate reductase in coronary artery disease. Circulation. 88:1463-1469.

16. Stipanuk, M.H. 1986. Metabolism of sulfur-containing amino acids. Ann. Rev. Nutr. 6:179-209.

17. Ubbink, J.B., W.J.H. Vermaak, A. Van der Merwe, and P.J. Becker. 1993. Vitamin B-12, vitamin B-6 and folate nutritional status in men with hyperhomocysteinemia. Am. J. Clin. Nutr. 57:47-53.

18. Selhub, J., P.F. Jacques, P.W.F. Wilson, D. Rush, and I.H. Rosenberg. 1993. Vitamin status and intake as primary determinants of homocysteinemia in an elderly population. J. Am. Med. Assoc. 270:2693-2698.

19. Pancharuniti, N., C.A. Lewis, H.E. Sauberlich, L.L. Perkins, R.C.P. Go, J.O. Ahvarez, M. Maculuso, R.T. Acton, R.B. Copeland, A.L. Cousins, et al. 1994. Plasma homocyst(e)ine, folate, and vitamin B12 concentrations and risk for early-onset coronary artery disease. Am. J. Clin. Nutr. 59:940-948.

20. Hopkins, P.N., L.L. Wu, S.C. Hunt, B.C. James, G.M. Vincent, and R.R. Williams. 1995. Higher plasma homocyst(e)ine and increased susceptibility to adverse effects of low folate in early familial coronary artery disease. Arterioscler. Thromb. Vasc. Biol. 15:1314-1320.

21. Kang, S., P.W.K. Wong, and M. Norusis. 1987. Homocysteinemia due to folate deficiency. Metab. Clin. Exp. 36:458-462.

22. Stabler, S.P., P.D. Maecell, E.R. Podell, R.H. Allen, G. Savage, and J. Lindenbaum. 1988. Elevation of total homocysteine in the serum of patients with cobalamin or folate deficiency detected by capillary gas chromatographymass spectrometry. J. Clin. Invest. 81:466-474.

23. Savage, D.G., J. Lindenbaum, S.P. Stabler, and R.H. Allen. 1994. Sensitivity of serum methylmalonic acid and total homocysteine determinations for diagnosing cobalamin and folate deficiencies. Am. J. Med. 96:239-246.

24. Kang, S., J. Zhou, P.W.K. Wong, J. Kowalisyn, and G. Strokosch. 1988. Intermediate homocysteinemia: a thermolabile variant of methylenetetrahydrofolate reductase. Am. J. Hum. Genet. 43:414-421.

25. Kang, S.S., P.W.K. Wong, H.G. Otto Bock, A. Horwitz, and A. Grix. 1991. Intermediate hyperhomocysteinemia resulting from compound heterozygosity of methylenetetrahydrofolate reductase mutations. Am. J. Hum. Genet. 48:546-551.

26. Brattström, L., B. Israelsson, F. Lingarde, and B. Hultberg. 1988. Higher total plasma homocysteine in vitamin B12 deficiency than in heterozygosity for homocystinuria due to cystathionine beta-synthase deficiency. Metab. Clin. Exp. 37:175-178.

27. Boers, G.H.J., B. Fowler, A.G.H. Smals, F.J.M. Trijbels, A.I. Leermakers, W.J. Kleijer, and P.W.C. Kloppenborg. 1985. Improved identification of heterozygotes for homocystinuria due to cystathionine synthase deficiency by the combination of methionine loading and enzyme determination in cultured fibroblasts. Hum. Genet. 69:164-169.

28. Wilcken, D.E.L., S.G. Reddy, and V.J. Gupta. 1983. Homocysteinemia, ischemic heart disease, and the carrier state for homocystinuria. Metab. Clin. Exp. 32:363-370.

29. Miller, J.W., J.D. Ribaya-Mercado, R.M. Russell, D.C. Shepard, F.D. Morrow, E.F. Cochary, J.A. Sadowski, S.N. Gershoff, and J. Selhub. 1992. Effect of vitamin B-6 deficiency on fasting plasma homocysteine concentrations. Am. J. Clin. Nutr. 55:1154-1160.

30. Ubbink, J.B., W.J.H. Vermaak, A. Van der Merwe, P.J. Becker, R Delport, and H.C. Potgieter. 1994. Vitamin requirements for the treatment of hyperhomocysteinemia in humans. J. Nutr. 124:1927-1933.

31. Brattström, L., B. Israelsson, B. Norrving, D. Bergqvist, J. Thorne, B. Hultberg, and A. Hamfelt. 1990. Impaired homocysteine metabolism in earlyonset cerebral and peripheral occlusive arterial disease. Atherosclerosis. 81:51-60.

32. Dudman, N.P.B., D.E.L. Wilcken, J. Wang, J.F. Lynch, D. Macey, and 
P. Lundberg. 1993. Disordered methionine/homocysteine metabolism in premature vascular disease. Its occurrence, cofactor therapy, and enzymology. $A r-$ terioscler. Thromb. 13:1253-1260.

33. Selhub, J., and J.W. Miller. 1992. The pathogenesis of homocysteinemia: interruption of the coordinate regulation by $S$-adenosylmethionine of the remethylation and transsulfuration of homocysteine. Am. J. Clin. Nutr. 55:131-138.

34. Park, Y.K., and H. Linkswiler. 1970. Effect of vitamin B6 depletion in adult man on the excretion of cystathionine and other methionine metabolites. J. Nutr. 100:110-116.

35. Shin, H.K., and H.M. Linkswiler. 1974. Tryptophan and methionine metabolism of adult females as affected by vitamin B-6 deficiency. J. Nutr. 104: 1348-1355.

36. Miller, J.W., M.R. Nadeau, D. Smith, and J. Selhub. 1994. Vitamin B-6 deficiency versus folate deficiency: comparison of responses to methionine loading in rats. Am. J. Clin. Nutr. 59:1033-1039.

37. Stabler, S.P., and R.H. Allen. 1994. Elevations of serum cystathionine and homocysteine in vitamin B-6, folate and cobalamin deficient rats. Blood. 84(Suppl. 1):118a. (Abstr.)

38. Ubbink, J.B., R. Delport, P.J. Becker, and S. Bissbort. 1989. Evidence of a theophylline-induced vitamin B6 deficiency caused by noncompetitive inhibition of pyridoxal kinase. J. Lab. Clin. Med. 113:15-22.

39. Ubbink, J.B., S. Bissbort, and W.J.H. Vermaak. 1990. Inhibition of pyridoxal kinase by methylxanthines. Enzyme. 43:72-79.

40. Ubbink, J.B., R. Delport, S. Bissbort, W.J.H. Vermaak, and P.J. Becker. 1990. Relationship between vitamin B-6 status and elevated pyridoxal kinase levels induced by theophylline therapy in humans. J. Nutr. 120:1352-1359.

41. Ubbink, J.B., W.J. Serfontein, and L.S. de Villiers. 1985. Stability of pyridoxal-5-phosphate semicarbazone: application in plasma vitamin B-6 analysis and population surveys of vitamin B-6 nutritional status. J. Chromatogr. 342: $277-284$

42. Araki, A., and Y. Sako. 1987. Determination of free and total homocys- teine in human plasma by HPLC with fluorescence detection. J. Chromatogr. 422:43-52.

43. Ubbink, J.B., W.J.H. Vermaak, and S. Bissbort. 1991. Rapid high-performance liquid chromatographic assay for total homocysteine levels in human serum. J. Chromatogr. 565:441-446.

44. Blanchard, J., S. Harvey, and W.J. Morgan. 1990. A rapid and specific high-performance liquid chromatographic assay for theophylline in biological fluids. J. Chromatogr. Sci. 28:303-306.

45. Allen, R.H., S.P. Stabler, D.G. Savage, and J. Lindenbaum. 1993. Metabolic abnormalities in cobalamin (vitamin B-12) and folate deficiency. FASEB (Fed. Am. Soc. Exp. Biol.) J. 7:1344-1352.

46. Conover, W.J. 1971. Practical Non-Parametric Statistics. John Wiley \& Sons, New York.

47. Brattström, L.E., B. Israelsson, J.O. Jeppson, and B.L. Hultberg. 1988

Folic acid: an innocuous means to reduce plasma homocysteine. Scand. J. Clin. Lab. Invest. 48:215-221.

48. Andersson, A., L. Brattström, and B. Israelsson. 1992. Plasma homocysteine before and after methionine loading with regard to age, gender, and menopausal status. Eur. J. Clin. Invest. 22:79-87.

49. Bostom, A.G., P.F. Jacques, M.R. Nadeau, R.R. Williams, R.C. Ellison, and J. Selhub. 1995. Post-methionine load hyperhomocysteinemia in person with normal fasting total plasma homocysteine: initial results from the NHLBI Family Heart Study. Atherosclerosis. 116:147-151.

50. Binkley, F., G.M. Christensen, and W.N. Jensen. 1952. Pyridoxine and the transfer of sulfur. J. Biol. Chem. 194:109-113.

51. Ubbink, J.B., W.J.H. Vermaak, A. van der Merwe, P.J. Becker, and H. Potgieter. 1995. Effective homocysteine metabolism may protect South African Blacks against coronary heart disease. Am. J. Clin. Nutr. 62:802-808.

52. Allen, R.H., S.P. Stabler, D.G. Savage, and J. Lindenbaum. 1993. Elevation of 2-methylcitric acid I and II levels in serum, urine, and cerebrospinal fluid of patients with cobalamin deficiency. Metab. Clin. Exp. 42:978-988. 